\title{
Research on network layout strategy of mobile opportunity perception in coal mines
}

\author{
Jingzhao Li and Zhi Xu* \\ School of Electrical Information and Engineering, \\ Anhui University of Science and Technology, \\ Huainan, China \\ Email: jzhli@, aust.edu.cn \\ Email: xu1995zhi@126.com \\ *Corresponding author
}

\begin{abstract}
The underground coal mine monitoring system has high costs of large-scale application, the network is not easy to expand, and it is difficult to achieve complete coverage of coal mines. To solve these problems, this paper presents a new data acquisition and transmission method based on 'wired + wireless', 'A, B, C and D class mobile node'. The method, combining the existing wired network and wireless network in the coal mine, using sparse heterogeneous converged networks centred on opportunistic networks, analyses the composition, function, connection law and historical information of four types of node and the system structure. Experimental results show that this method can further realise the dynamic planning of the node interaction path. Then, it predicts and corrects the data of artificial mobile nodes and fixed interaction information in the next cycle. Finally, it achieves a powerful guarantee for internet of things, interaction of objects, intelligent perception, and intelligent processing in the mine safety management.
\end{abstract}

Keywords: coal mine underground; opportunity perception; mobile network; mobile nodes; sensory nodes; information interaction; heterogeneous network; intelligent perception; dynamic path; safety management.

Reference to this paper should be made as follows: Li, J. and Xu, Z. (2020) 'Research on network layout strategy of mobile opportunity perception in coal mines', Int. J. Computational Science and Engineering, Vol. 22, No. 1, pp.124-134.

Biographical notes: Jingzhao Li received his $\mathrm{PhD}$ from the Hefei University of Technology, Anhui, in 2003. He is a Professor at the Anhui University of Science and Technology, China. His current research interests include the internet of things and computer monitoring technology.

Zhi Xu received his Bachelor degree from the Anhui University of Science and Technology in 2018, China. Currently, he is an MS degree candidate at the College of Electrical Information and Engineering, AUST. His current research interests are the internet of things and embedded system.

\section{Introduction}

Industrial and mining enterprises generally have wired networks such as industrial Ethernet, which collect and transmit industrial field data at critical locations or locations to realise remote monitoring of enterprise production. Due to the cost and other reasons, the layout of the wired network is relatively limited, resulting in less information collected and transmitted, and it is difficult to further improve the information production and management level of enterprises. If the wired network + wireless LAN node method is adopted, it requires too many nodes, which is not only costly but also inconvenient to manage; if a wireless extensive area network is used to set and manage data collection and communication nodes, since the current wireless extensive area network is charged by traffic or time, there is a burden to the company for long-term use.

Coal mine safety production needs to monitor coal mine underground coal mining, excavation, transportation, lifting, ventilation, drainage, power supply, pressure supply, safety supervision, and other aspects. Because the underground mine roadway is narrow and long, only a few metres wide and tens of kilometres long, the information perception is significantly different from the surface. At present, the self-organising wireless network is applied to the coal mine environment, to monitor the downhole environment and equipment parameters. The following problems also exist:

1 The network construction cost is high: the coal mine has a long extension distance and a large range. Due to the narrow structure of the underground tunnel, it is usually necessary to densely deploy a large number of nodes to ensure network coverage and connectivity. The cost of those nodes is high in large-scale applications. Because sensor nodes are limited in energy, storage and computing power (Borgia, 2014), traditional wireless sensor networks are difficult to expand and so on. It is impossible to achieve complete 
coverage of coal mines (Zhang et al., 2018; Xu et al., 2017).

2 Network transmission performance is low: due to the long and narrow structure of the mine roadway, the mine roadway has a cable that passes a large current. Due to the interference of these electromagnetic waves, the wireless signal transmission has a multipath effect. Therefore, the transmission distance becomes shorter, and the traditional wireless network routing algorithm has poor transmission performance under the coal mine (Zhao et al., 2013).

3 Not easy to maintain: in a fixed deployment sensor network, the data flow follows a many-to-one mode. Some sensor nodes, such as sink nodes and nodes around the sink node, need to bear more communication load. It is easy to exhaust their energy prematurely and cause the energy hole problem. Due to the humid environment in the coal mine and the corrosive substances in the mine, the nodes in the mine environment are easily corroded, and a large number of nodes are difficult to maintain.

4 Poor scalability: as coal mines continue to mine and the monitoring range increases, traditional wireless self-organising networks are not easy to expand. Because of the special environment of the mine, it is difficult to arrange nodes in a large-scale manner in the underground coal mine. It is difficult to meet the requirements of physical interconnection and intelligent linkage only by wired network and fixed nodes.

At present, there are few wired and wireless heterogeneous network nodes in coal mines, which can only achieve local coverage. It is difficult to achieve comprehensive sensing and effective transmission of mine information. Therefore, this paper proposes to apply the opportunity network technology in the coal mine environment, combined with the existing wired network and wireless network in the coal mine. Using dynamic sensing technology and underground mobile equipment, mining vehicles and personnel to build mobile opportunity sensor network. Establishing the sparse heterogeneous converged network with the core of the opportunity network realises the comprehensive sensing and reliable transmission of mine information at low cost (Trifunovic et al., 2017).

\section{Related work}

Opportunity mobile sensing technology provides a new perception model for coal mine IoT and brings a series of new challenges. In the opportunity network, due to the movement of nodes, sparse network, and signal attenuation, there is no guarantee that there is a complete communication path between the source node and the destination node. The network and links are disconnected and reconnected frequently in this environment. The routing protocols of traditional wireless ad hoc networks are not suitable for working in opportunistic networks (Zhou and
Peng, 2018). In recent years, research on the application of opportunity network routing protocols has been the focus of scholars at home and abroad (Zhang et al., 2014). The Institute of Information Engineering of the Chinese Academy of Sciences researched mobile delay network and opportunistic network theory and key technologies. Based on the two application fields of vehicle network and ocean monitoring, the mobile model, architecture, transmission technology and enhancement mechanism of mobile delay network various aspects have been studied. The urban vehicle movement model and the general mobile network architecture (Wu et al., 2012; Liu et al., 2010) are proposed. Since the transmission opportunities of the opportunistic network depend on the node movement. The researchers propose many typical mobile models by studying the motion laws of nodes in different application scenarios, and analyse the relationship between the mobile model and network performance. Kerdsri and Veeraklaew (2015) studied and analysed the relationship between energy consumption and mobile models of mobile nodes. Nguyen et al. (2015) studied the mobility of humans and the impact on the performance of opportunistic networks and proposed the opportunity network and map-based opportunistic network of well-known small world structures under sufficient conditions. Batabyal and Bhaumik (2015) try to understand the mobility of humans, vehicles and wildlife; the researchers collected movement trajectories, proposed moving models, and analysed different movement parameters, proposed different movement models, and studied mobility to the impact of opportunity routing performance. Pásztor et al. (2007) proposed to provide a mobile model for the ZebraNet project through the use of COOJA simulators to achieve random selection-based propagation. Due to the motion of the nodes, the topology of the network will change constantly. There may be no end-to-end connections and scarce resources, making the traditional routing algorithm unsuitable. The study believes that opportunistic routing is dynamically generated, and any node can become the next hop as long as the destination node.

For an opportunistic network with less infrastructure, Dhurandher et al. (2013) proposed a prediction-based routing that uses the behavior information of the nodes to find the best routing protocol for the next node. Soares et al. (2014) proposed a scheme called Geospray VDTN routing protocol based on geographic data and the scheme between multi-copy and single-copy of the hybrid method. Compared to traditional positioning and non-positioning single copy and multiple copy routing protocols, Geospray significantly improved delivery rate and reduced Transmission delay. For wired and wireless heterogeneous network transmission problems, Li et al. (2014) proposed a reliable multicast protocol, which effectively improves network throughput by establishing coding in opportunistic routing and random linear networks. Opportunity perception is a new mode of perception and transmission. How to use mobile personnel and vehicles to establish reasonable mobile and auxiliary node deployment, meet the sensing 
and transmission requirements of the entire application environment. It faces the problem of the coverage of existing networks and the requirement of the perception network. For the opportunity network coverage problem, the researchers conducted research on coverage quality measurement, liquidity model evaluation, and network deployment plan design (Zhao et al., 2015). Ferretti (2013) discusses the identification and management of overlay topologies in a tracking-driven opportunistic network. It uses different types of data sets, and experimental evaluations confirm the feasibility of the proposed method. Sitanayah et al. (2010) proposed a distributed low-density boundary connection method for randomly deployed wireless sensor networks by studying a large number of sensing layer nodes, which solved the problem of sparse network information transmission. Qin et al. (2015) introduced cloud services to improve network coverage and provide network connectivity protection.

Applying the opportunity movement perception to the mine environment has the following technical advantages:

1 The network deployment cost is lower, and the network is easier to maintain. The mobile node is deployed in the underground mobile platform, such as equipment, mining vehicles, personnel, etc., and the underground network can be used to complete the underground network with a small number of fixed nodes. The deployment is simple, the cost is low, and the maintenance is convenient (Yang et al., 2016).

2 The mobility of nodes can promote perceived coverage and data transmission. On the one hand, the nodes can periodically or randomly reach the various places in the coal mine with the mobile platform, and these nodes can sense the environment information anytime and anywhere; on the other hand, these nodes use the opportunity due to the mutual contact between the mobile nodes. The transmission mode transmits the sensing data in the intermittently connected network environment, which solves the routing hole problem of the traditional wireless self-organising network ( $\mathrm{Li}$ et al., 2017).

3 The system is more scalable. With the mining of coal mines, the nodes will advance along with the mobile platform carrying the nodes. Only a small number of nodes can be added to meet the expansion of the system application scale and achieve full coverage of the coal mine network (Wang et al., 2015).

Therefore, this project is aimed at the mine environment wth a limited narrow roadway, enormous sense and control information, and proposes a heterogeneous fusion network model of wired node + fixed wireless node + mobile wireless node, and a new heterogeneous network model for coal mine environment. Key theories and technologies such as mobile node mobility model and heterogeneous network routing algorithm are studied to construct a new heterogeneous converged network with unique characteristics of mine environment (Sasirekha et al., 2015).

\section{New opportunity-aware network thinking}

Based on sufficient survey and communication with production and management personnel of industrial and mining enterprises, this paper proposes a new method for data acquisition and transmission of the fixed site + mobile node that is used in the enterprise construction site). The method sets four types of nodes A, B, C and D according to the underground coal mine production environment. Class A and class B are fixed nodes that are sparsely arranged in key locations in coal mines. They can detect the environment and main equipment conditions of the nodes. Class A nodes directly access the underground optical fibre Ethernet, which can surround the environment. Comprehensive information and device status are transmitted directly to the ground monitoring centre by optical fibre Ethernet, while class B nodes are not connected to downhole optical fibre Ethernet. Both C-type nodes and D-type nodes are mobile nodes. Use their mobility to monitor the entire downhole environmental parameters, and communicate with the class $\mathrm{B}$ nodes to obtain comprehensive information and equipment status of the environment around the class B nodes. Then, the above information will be passed to class A nodes by moving to class A nodes. Its structure is shown in Figure 1.

Figure 1 Coal mine underground opportunity network system structure diagram (see online version for colours)

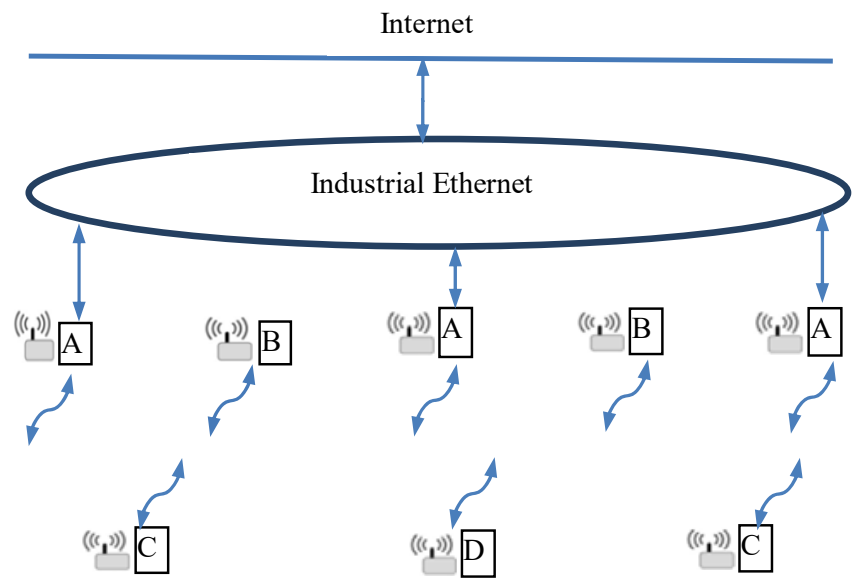

A class A node is a fixed node. It has the following functions:

1 wired network communication

2 wireless network communication

3 main equipment and environment and other parameters are collected, which are expressed by node A.

It is mainly composed of three parts, a sensing module, a microprocessor module, and a wireless communication 
module. The sensing module is responsible for collecting the environmental parameters around the node and the main state of the node area device; the wireless communication module mainly accepts the information sent by the class $\mathrm{C}$ and class D mobile nodes; the microprocessor module is the control core, which processes the above information and passes through the underground industry Ethernet to transmit to the ground. The composition of class A nodes is shown in Figure 2.

Figure 2 Class a node structure (see online version for colours)

\begin{tabular}{|c|c|c|c|}
\hline $\begin{array}{c}\text { Mobile } \\
\text { node }\end{array}$ & Class A node & $\begin{array}{c}\text { Wire } \\
\text { communication }\end{array}$ & $\begin{array}{c}\text { Industrial } \\
\text { Ethernet }\end{array}$ \\
\hline $\begin{array}{c}\text { Wireless } \\
\text { communicatio }\end{array}$ & Sensor & $\begin{array}{c}\text { Major } \\
\text { equipment }\end{array}$ \\
\hline & Sensor & $\begin{array}{c}\text { Environmental } \\
\text { parameters }\end{array}$ \\
\hline
\end{tabular}

Class B nodes are also fixed nodes. Its functions are as follows:

1 only wireless communication, no wired communication

2 general equipment and environment sensor parameters collection, which are expressed by node B.

The class B node is mainly composed of three parts, a sensing module, a microprocessor module, and a wireless communication module. The sensing module is responsible for collecting environmental parameters around the node and the main state of the node area device; the wireless communication module interacts with the $\mathrm{C}$ class and $\mathrm{D}$ class mobile nodes; the microprocessor module is the control core, and the information collected by the class $\mathrm{B}$ node is processed and passed. Class $\mathrm{C}$ and class D mobile nodes are then sent to class A nodes. The composition of class B nodes is shown in Figure 3.

Figure 3 Class B node structure (see online version for colours)

\begin{tabular}{|c|c|c|c|c|}
\hline $\begin{array}{c}\text { Mobile } \\
\text { node }\end{array}$ & Class B node & Sensor & $\begin{array}{c}\text { Major } \\
\text { equipment }\end{array}$ \\
\hline $\begin{array}{c}\text { Wireless } \\
\text { communication }\end{array}$ & $\leftrightarrow$ & Sensor & $\begin{array}{c}\text { Environmental } \\
\text { parameters }\end{array}$ \\
\hline
\end{tabular}

A class $\mathrm{C}$ node is a mobile node. It is a transport vehicle or a node carried on the installation or staff that is frequently flowed or moved on the production site of the enterprise, and does not require a mobile device or personnel specially added for communication. It has the following characteristics:

1 wireless communication function

2 environmental parameter collection function, which is expressed by node $\mathrm{C}$.

The dynamic monitoring of the entire downhole environment parameters is realised by the mobility of the C-type nodes, and at the same time, it can communicate with the B-type nodes to obtain comprehensive information and equipment status of the environment around the B-type nodes, and finally move to the A-type node. Moreover, interact with it to transfer the above information to the class A node. The composition of the $\mathrm{C}$ class node is shown in Figure 4.

Figure 4 Class C node structure (see online version for colours)

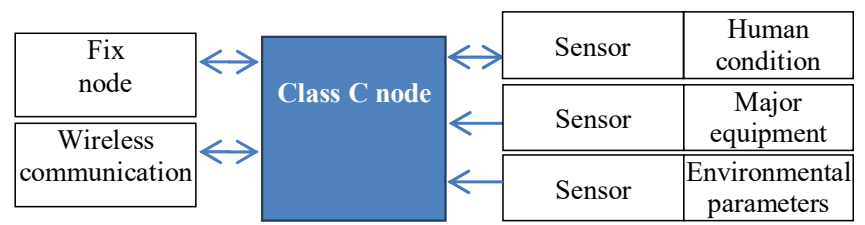

A class D node is also a mobile node. The mobile node that is artificially set or added to achieve better network functions has functions similar to that of the $\mathrm{C}$ class. The difference is that it is explicitly added to improve network communication and field data collection capabilities, and is represented by node $\mathrm{D}$. The $\mathrm{D}$ class node composition is shown in Figure 5.

Figure 5 Class D node structure (see online version for colours)

\begin{tabular}{|c|c|c|c|c|}
\hline $\begin{array}{c}\text { Fix } \\
\text { node }\end{array}$ & Class D node & Sensor & $\begin{array}{c}\text { Human } \\
\text { condition }\end{array}$ \\
\hline $\begin{array}{c}\text { Wireless } \\
\text { communication }\end{array}$ & $\leftrightarrow$ & Sensor & $\begin{array}{c}\text { Environmental } \\
\text { parameters }\end{array}$ \\
\hline
\end{tabular}

The functions of the above four types of nodes are shown in Table 1.

Table 1 Characteristics and functions of four types of nodes

\begin{tabular}{lcccc}
\hline Functionltype & Node $A$ & Node B & Node C & Node D \\
\hline Wired network & $\mathrm{Y}$ & $\mathrm{N}$ & $\mathrm{N}$ & $\mathrm{N}$ \\
Wireless network & $\mathrm{Y}$ & $\mathrm{Y}$ & $\mathrm{Y}$ & $\mathrm{Y}$ \\
Fixed node & $\mathrm{Y}$ & $\mathrm{Y}$ & $\mathrm{N}$ & $\mathrm{N}$ \\
Move node & $\mathrm{N}$ & $\mathrm{N}$ & $\mathrm{Y}$ & $\mathrm{Y}$ \\
Original & $\mathrm{Y}$ & $\mathrm{Y}$ & $\mathrm{Y}$ & $\mathrm{N}$ \\
Add & $\mathrm{N}$ & $\mathrm{N}$ & $\mathrm{N}$ & $\mathrm{Y}$ \\
\hline
\end{tabular}

\section{Node history information analysis}

Through research, we found that mobile devices or personnel in the underground coal mines are not entirely moving randomly, and their movement tends to a certain degree to some extent. If a mobile node carried by an opportunistic mobile device or a person frequently meets a particular fixed node in a certain period in the past, there is a high possibility that they will meet in the future. In the industrial field, mobile devices are generally doing the pan-cycle movement, and a mobile device (or personnel) equipped with (or with) a mobile node may pass a fixed node at the same time at different stages on a fixed place. Therefore, the analysis of the connection rules between nodes and their historical information will help to improve the communication efficiency of the industrial network mobile opportunity network in the next cycle (or phase).

Let the number of nodes of the first type of fixed node node A be $a$, denoted by $i=1,2, \ldots, a$; the number of nodes of the second type of fixed node node $\mathrm{B}$ is $b$, with $j=1,2$, 
$\ldots, b$. To indicate; set its activity period to $T$ (generally expressed in days). Each cycle is divided into $U$ time units according to the nature of the work, such as shifts, and $\mathrm{Z}=\mathrm{T} * \mathrm{U}(\mathrm{m}=1,2, \ldots, Z)$ is the recorded historical time unit number.

The element $A_{i}(k, m)$ is used to indicate the number of times the $i^{\text {th }}$ fixed node in node A interacts with the $k^{\text {th }}$ mobile node in node $\mathrm{C}$ in $m$ time units, and in the last $z$ time units, some in node A. The mobile node historical interaction information of all fixed nodes $i$ and node $\mathrm{C}(k=$ $1,2, \ldots, c)$ is stored in $A_{i}$, as shown in equation (1).

$$
A_{i}=\left[\begin{array}{l}
A_{i}(1,1), \ldots A_{i}(1, m), \ldots A_{i}(1, z) \\
\ldots \\
A_{i}(k, 1), \ldots A_{i}(k, m), \ldots A_{i}(k, z) \\
\ldots \\
A_{i}(c, 1), \ldots A_{i}(c, m), \ldots A_{i}(c, z)
\end{array}\right], i=1,2, \ldots, a
$$

The element $B_{j}(k, m)$ is used to indicate the number of times that the $j^{\text {th }}$ fixed node in node B interacts with the $k^{\text {th }}$ mobile node in node $\mathrm{C}$ in $m$ time units, and in the last $z$ time units, some in node $\mathrm{B}$. The mobile node historical interaction information of all fixed nodes $j$ and node $\mathrm{C}(k=1,2 . \ldots, c)$ is stored in $B_{j}$, as shown in equation (2).

$$
B_{j}=\left[\begin{array}{l}
B_{j}(1,1), \ldots B_{j}(1, m), \ldots B_{j}(1, z) \\
\ldots \\
B_{j}(k, 1), \ldots B_{j}(k, m), \ldots B_{j}(k, z) \\
\ldots \\
B_{j}(c, 1), \ldots B_{j}(c, m), \ldots B_{j}(c, z)
\end{array}\right], j=1,2, \ldots, b
$$

The element $C_{i}(k, m)$ is used to indicate the number of times the $i^{\text {th }}$ fixed node in node A interacts with the $k^{\text {th }}$ mobile node in node $\mathrm{D}$ in $m$ time units, and in the last $z$ time units, one in node $\mathrm{A}$. The mobile node historical interaction information of all fixed nodes $i$ and node $\mathrm{D}(k=1,2, \ldots, b)$ is stored in $C_{i}$, as shown in equation (3).

$$
C_{i}=\left[\begin{array}{l}
C_{i}(1,1), \ldots C_{i}(1, m), \ldots C_{i}(1, z) \\
\ldots \\
C_{i}(k, 1), \ldots C_{i}(k, m), \ldots C_{i}(k, z) \\
\ldots \\
C_{i}(b, 1), \ldots C_{i}(b, m), \ldots C_{i}(b, z)
\end{array}\right], i=1,2, \ldots, c
$$

Similarly, the element $D_{j}(k, m)$ is used to indicate the number of times the $i^{\text {th }}$ fixed node in node $\mathrm{B}$ interacts with the $k^{\text {th }}$ mobile node in node $\mathrm{D}$ in $m$ time units, and in the last $z$ time units, nodeB In a fixed node $i$ and all $b$ in node D $(k=1,2, \ldots, b)$ mobile node historical interaction information is stored in $\mathrm{Dj}$, As shown in equation (4).

$$
D_{j}=\left[\begin{array}{l}
D_{j}(1,1), \ldots D_{j}(1, m), \ldots D_{j}(1, z) \\
\ldots \\
D_{j}(k, 1), \ldots D_{j}(k, m), \ldots D_{j}(k, z) \\
\ldots \\
D_{j}(c, 1), \ldots D_{j}(c, m), \ldots D_{j}(c, z)
\end{array}\right], j=1,2, \ldots, b
$$

\section{The fourth type of mobile node prediction in the next cycle}

\subsection{The next cycle of manual mobile node interaction with fixed information $T_{y}^{\prime}$ prediction}

To simplify the following analysis, the third type of mobile node analysed above is combined with the first type of fixed history information, and the third type of mobile node and the second type of fixed node history information, represented by a matrix $T_{x}$. As shown in equation (5).

$$
T_{x}=\left[\begin{array}{l}
T_{x}(1,1), \ldots T_{x}(1, m), \ldots T_{x}(1, z) \\
\ldots \\
T_{x}(k, 1), \ldots T_{x}(k, m), \ldots T_{x}(k, z) \\
\ldots \\
T_{x}(c, 1), \ldots T_{x}(c, m), \ldots T_{x}(c, z)
\end{array}\right], x=1,2 \ldots a+b
$$

Combining the fourth type of mobile node analysed above with the first type of fixed history information, and the history information of the fourth type of mobile node and the second type of fixed node, represented by a matrix $T_{y}$ as shown in equation (6), The next cycle of expected information interaction is represented by $T_{y}^{\prime}$ as shown in equation (7).

$$
\begin{aligned}
T_{y} & =\left[\begin{array}{l}
T_{y}(1,1), \ldots T_{y}(1, m), \ldots T_{y}(1, z) \\
\ldots \\
T_{y}(k, 1), \ldots T_{y}(k, m), \ldots T_{y}(k, z) \\
\ldots \\
T_{y}(c, 1), \ldots T_{y}(c, m), \ldots T_{y}(c, z)
\end{array}\right], y=1,2 \ldots a+b \\
T_{y}^{\prime} & =\left[\begin{array}{l}
T_{y}^{\prime}(1,1), \ldots T_{y}^{\prime}(1, m), \ldots T_{y}^{\prime}(1, z) \\
\ldots \\
T_{y}^{\prime}(k, 1), \ldots T_{y}^{\prime}(k, m), \ldots T_{y}^{\prime}(k, z) \\
\ldots \\
T_{y}^{\prime}(c, 1), \ldots T_{y}^{\prime}(c, m), \ldots T_{y}^{\prime}(c, z)
\end{array}\right], y=1,2 \ldots a+b
\end{aligned}
$$

It is assumed that all fixed nodes and mobile node information expected in the next cycle are represented by a matrix $T_{g}^{\prime}$ as shown in equation (8).

$$
T_{g}^{\prime}=\left[\begin{array}{l}
T_{g}^{\prime}(1,1), \ldots T_{g}^{\prime}(1, m), \ldots T_{g}^{\prime}(1, z) \\
\ldots \\
T_{g}^{\prime}(k, 1), \ldots T_{g}^{\prime}(k, m), \ldots T_{g}^{\prime}(k, z) \\
\ldots \\
T_{g}^{\prime}(b, 1), \ldots T_{g}^{\prime}(b, m), \ldots T_{g}^{\prime}(b, z)
\end{array}\right], g=1,2 \ldots a+b
$$

Construct an error function e as shown in equation (9).

$$
e=\left[T_{g}^{\prime}-T_{x}-T_{y}\right]^{2}
$$

At the same time, the error function $E$ is obtained as shown in equation (10). 


$$
E=\min \left(\sum_{1}^{x} e\right)
$$

From equation (10), the next period of manual mobile node interaction with fixed information $T_{y}^{\prime}(k)$ can be obtained.

Node interaction state matrix $T_{y}^{\prime}(k)$ calculates pseudocode:

1 input error0, $E=0$

2 calling the history information $T_{x}[k]$ from the database of the third type of mobile node and the fixed node

3 calling the history information of the fourth type of mobile node and the fixed node, $T_{y}[k]$ from the database

4 calculate $e=\left(T_{g}^{\prime}(k)-T_{x}[k]-T_{y}[k]\right) *\left(T_{g}^{\prime}(k)-T_{x}[k]-T_{y}[k]\right)$.

$5 \quad E=E+e$

6 If $E<=$ error0?, return 2, else, go on

7 output: $T_{y}^{\prime}(k)$.

\subsection{Kalman node interaction prediction model}

\subsubsection{Establishing the Kalman node interaction system equation}

$T_{y}^{\prime}$ is also affected by the underground coal environment, production status, production efficiency, and other information. The Kalman filter is improved, and the parameters are predicted to establish an interaction model based on improved Kalman movement and fixed nodes. Thereby obtaining a more accurate manual mobile node and fixed node monitoring information $T_{y}^{\prime \prime}$.

The system state equation of the Kalman filter is established according to the underground coal environment, production status, production efficiency, and other information, as shown in equation (11).

$$
Q(k+1)=L(k) Q(k)+\omega(k)
$$

In equation (11): $Q(k)$ is the interaction information between the mobile node and the fixed node, which is a system matrix and is a system interference signal whose system obeys a Gaussian distribution, which is represented by equation (12).

$$
\omega(k)=\left[w_{k}^{1}, w_{k}^{2}, \ldots, w_{k}^{a+b}\right]^{T} ; \omega(k) \in N(0, J)
$$

$J$ is the covariance of the interference signal of coal mine underground environment, production status, production efficiency, etc., which can be represented by formula (13).

$$
J=\operatorname{Covar}[\omega(k)]=E\left[\omega(k) \cdot \omega(k)^{T}\right]
$$

Next, we reconstruct the state observation equation of the moving and fixed nodes as in equation (14).

$$
s(k)=H(k) Q(k)+v(k)
$$

In equation (14), for the observation matrix of the moving and fixed nodes, $H(k)$ is the state matrix of the moving and fixed nodes. The enthalpy $(k)$ in the formula (14) is a downhole environment, a production condition, and a production efficiency observation noise signal obeying a Gaussian distribution, as shown in the formula (15).

$$
v(k)=\left[v_{k}^{1}, v_{k}^{2}, \ldots, v_{k}^{a+b}\right]^{T} ; v(k) \in N(0, R)
$$

The covariance $R$ of the moving and fixed nodes under the underground environment, production status, and production efficiency observation interference information is as shown in equation (16).

$$
R=\operatorname{Covar}[v(k)]=E\left[v(k) \cdot v(k)^{T}\right]
$$

It is assumed that the downhole environment, production conditions, production efficiency interference signal $\omega(k)$ and state observation noise signal $v(k)$ do not change with the parameter state such as the activity period. Below we combine the downhole environment, production status, production efficiency interference signal, and observer noise signal. Moreover, its covariance to estimate the interaction state between the mobile node and the fixed node.

\subsubsection{Kalman node interaction state prediction and correction}

The improved Kalman filter algorithm for node interaction estimation is divided into two steps: prediction and correction.

- First step: node interaction state prediction

The information model of the underground environment, production status, and production efficiency is used to predict the node interaction status of the next activity cycle.

Assuming that the current state is $K$, the previous state is $(K-1)$, and the current node interaction state is predicted $Q(k \mid k-1)$ as shown in equation (17).

$Q(k \mid k-1)=L(k-1) Q(k-1 \mid k-1)$

Let $P(k \mid k-1)$ be the covariance of $Q(k \mid k-1)$, then $P(k \mid k-1)$ is expressed by equation (18).

$$
P(k \mid k-1)=L(k-1) P(k-1 \mid k-1) L^{T}(k-1)+J
$$

- Second step: node interaction state correction

Above, we have already predicted the current downhole environment, production status, and production efficiency information model state and have prediction results. Next, we will correct the prediction result based on the current state detection value to obtain the optimal state $Q(k)$. The estimated $Q(k \mid k)$ is as shown in equation (19).

$$
Q(k \mid k)=Q(k \mid k-1)+K(k)[s(k)-H(k) Q(k \mid k-1)]
$$


In equation (19), $K(k)$ is a Kalman gain matrix whose main function is to correct the state, which can be obtained by equation (20).

$$
\begin{aligned}
K(k)= & P(k \mid k-1) H^{T}(k) \\
& {\left[H(k) P(k \mid k-1) H^{T}(k)+R\right]^{-1} }
\end{aligned}
$$

So far, we have obtained the optimal estimate $Q(k)$ of the current state $Q(k \mid k)$.

To keep the system running, it is also necessary to calculate the covariance $P(k \mid k)$ of the optimal estimate $Q(k \mid k)$ of $Q(k) . P(k \mid k)$ can be obtained by formula (21).

$$
P(k \mid k)=[I(k)-K(k) H(k)] P(k \mid k-1)
$$

In formula (21), $I(k)$ is an identity matrix of 1 . When the system enters the $P(k \mid k+1)$ or $(k+1)$ state, $P(k \mid k)$ is the $P(k \mid k-1)$ of the equation (21). In this way, $P(k \mid k)$ can proceed from the regression operation.

\subsubsection{Convergence analysis of Kalman node interaction state}

In the above regression operation, the error of the error function $E$ of the system will be continuously accumulated. When returning to a certain number of steps, the algorithm may diverge. To ensure the convergence of the above algorithm, the author introduces a desalination factor inversely proportional to the time by repeated argumentation and comparison.

Equation (21) is modified as shown in equation (22)

$$
P(k \mid k-1)=\alpha(k) L(k-1) P(k-1 \mid k-1) L^{T}(k-1)+J
$$

In formula (22), $\alpha(k)$ is a forgetting factor for improving the accuracy of the Kalman filter.

Given the estimated values of the initial state of the current downhole environment, production conditions, and production efficiency information, the prediction and correction iteration calculations are performed according to equations (17)-(22), and the system accuracy parameter $\varepsilon$ is set in advance, when the system operation satisfies the equation (23). When the operation ends, the more accurate information $Q^{\prime}$ will be obtained.

$$
\sqrt{Q^{\prime 2}(k \mid k)+Q^{\prime 2}(k \mid k-1)} \leq \varepsilon
$$

Substitute it into equation (24), you can calculate

$$
T_{y}^{\prime \prime}=T_{y}^{\prime}(k)+\left[Q(k)-Q^{\prime}(k)\right] T_{y}(k)
$$

$T_{y}^{\prime \prime}(k)$ is the next cycle of the manual mobile node and fixed information interaction matrix.

\subsubsection{Kalman node interaction state prediction and correction algorithm implementation}

Kalman node interaction state estimation and correction algorithm pseudo code are as follows:
$1 \quad$ Input: error $1, T, k$, steps, $H(k), Q(k), T_{y}^{\prime}(k)$

2 call Omiga $W$ and Obersive $U$ from the database

3 calculate the covariance $J$ and $R$

4 calculating the observation matrix $S(k)$

5 calculate the prediction matrix $Q(k \mid k-1)$

6 calculate the covariance $P(k \mid k)$;

7 correct the covariance $P(k \mid k-1)$;

8 calculating a Kalman gain matrix

9 calculate the optimal estimate $Q^{\prime}(k \mid k)$;

$10 E_{1}=$ calculation SQRT $Q^{\prime}(k \mid k) * Q^{\prime}(k \mid k)+Q^{\prime}(k \mid k-1)$ * $\left.Q^{\prime}(k \mid k-1)\right)$

11 if $E_{1}<=$ error1?, return 3, else, go on

12 output: $T_{y}^{\prime \prime}(k)$.

\section{Dynamic route of the fourth type of node}

Above, we have obtained the interaction information of the system nodes. The dynamic programming idea is applied below to plan the path of the node interaction.

Let the nodes of the first type fixed node nodeA and the second type fixed node nodeB be $X=\left[x_{1}, x_{2}, \ldots, x_{a+b}\right]^{\mathrm{T}}$, and the number of the fourth type mobile node nodeD is $Y=\left[y_{1}, y_{2}, \ldots, y_{l}\right]^{\mathrm{T}}$, obtain the equation of state as shown in equation (25)

$$
\left[\begin{array}{c}
x_{1} \\
x_{2} \\
\cdot \\
\cdot \\
x_{a+b}
\end{array}\right]_{y}^{\prime}=\left[\begin{array}{l}
A_{i}(1,1), \ldots A_{i}(1, m), \ldots A_{i}(1, z) \\
\ldots \\
A_{i}(k, 1), \ldots A_{i}(k, m), \ldots A_{i}(k, z) \\
\ldots \\
A_{i}(b, 1), \ldots A_{i}(b, m), \ldots A_{i}(b, z)
\end{array}\right] \cdot\left[\begin{array}{c}
y_{1} \\
y_{2} \\
\cdot \\
\cdot \\
y_{l}
\end{array}\right]
$$

To make writing simple, use equation (26).

$$
X=T_{y}^{\prime \prime} \cdot Y
$$

Next, we also need to find the relationship between the fourth type of mobile node and the fixed node and obtain the equations (27) and (28) by inverting $T_{y}^{\prime \prime}$.

$$
\begin{aligned}
& Y=T_{y}^{\prime \prime-1} \cdot X \\
& {\left[\begin{array}{c}
y_{1} \\
y_{2} \\
\cdot \\
\cdot \\
y_{l}
\end{array}\right]=\left[\begin{array}{l}
i A_{i}(1,1), \ldots i A_{i}(1, m), \ldots i A_{i}(1, z) \\
\ldots \\
i A_{i}(k, 1), \ldots i A_{i}(k, m), \ldots i A_{i}(k, z) \\
\ldots \\
i A_{i}(b, 1), \ldots i A_{i}(b, m), \ldots i A_{i}(b, z)
\end{array}\right] \cdot\left[\begin{array}{c}
x_{1} \\
x_{2} \\
\cdot \\
\cdot \\
x_{a+b}
\end{array}\right]}
\end{aligned}
$$

Let $T_{y}^{\prime \prime-1}=H$, and consider the constraint $U$, then get the equation (29).

$$
Y^{\prime}=H X+U
$$


Its general expression is as shown in equation (30).

$$
Y^{\prime}=f(X, U, T)
$$

Equation (30) is discretised as shown in equation (31).

$$
Y_{k}^{\prime}=F\left[X_{k}, U_{k}\right]
$$

In formula (31), $k=0,1, \ldots, Z$. Constructing an energy function of equation (32) under the condition that the constraint $G[X, U]<=0$.

$$
J=\Phi[X]+\Sigma L[X, U]
$$

In equation (32), $\Phi[X]$ is the terminal state of the planned path

Let us look for the optimal path in the case where the energy function $E$ is minimised under the above constraints and states.

Obviously, in the case where the given constraint $U$ and the original state (fixed node state) are known, the path $Y$ can be obtained from equation (27) or equation (28).

This system is a multi-segment optimal planning decision. The decision must be made piece by piece, and the optimal plan is completed so that the energy function $E$ is a minimum value.

For a multi-segment optimal decision problem, regardless of the selection at the beginning stage, the state sequence and control sequence of each segment after k must be an optimal control sequence for the $Z-k$ segment, which should make the $Z-k$ term after the energy function $\mathrm{E}$ extremely small, which is

$$
\begin{aligned}
& E\left[Y_{N}-1\right]=\min \ldots \min \left(\Phi\left[X_{N}\right]+\Sigma L\left[X_{k}, U_{k}\right]\right) \\
& E\left[Y_{N}\right]=\min \left(E\left[Y_{N}-1\right]+\Sigma L\left[X_{0}, U_{0}\right]\right) \\
& Y_{0}=F\left[X_{0}, U_{0}\right]
\end{aligned}
$$

Calculated according to equations (25)-(32) and recursed according to equations (33)-(35), the optimal plan $\mathrm{Y}^{\prime}$ can be obtained.

Its pseudocode is as follows:

Node interaction optimal dynamic path planning algorithm pseudo code:

1 input: $X, T, k$, steps, $U, Y_{\mathrm{pxl}}$

2 storage data into a database

$3 Y_{0}=F\left[X_{0}, U_{0}\right]$, calculate $Y_{0}$

4 the calculation result of calling program $1 T_{y}^{\prime \prime}(k)$

5 performing matrix inversion on $T_{y}^{\prime \prime}(k)$ to obtain $H$

6 apply $Y^{\prime}=H X+U$ to find $Y^{\prime}$

7 substituting $E\left[Y_{N}-1\right]=\min . . . \min \left(\Phi\left[X_{N}\right]+\Sigma L\left[X_{k}, U_{k}\right]\right)$

8 calculate $E\left[Y_{N}\right]=\min \left(\mathrm{E}\left[Y_{N}-1\right]+\Sigma L\left[X_{0}, U_{0}\right]\right)$

9 if $E\left[Y_{N}\right]<=Y_{p x l}$ ?, return 7, else, go on

10 output: $Y^{\prime}$

\section{Experiments and results analysis}

\subsection{Experimental device and environment}

The class A node is mainly composed of STM32F103ZET6 MCU, Ethernet interface module, wireless communication LoRa module, an environment sensing module, device parameter sensing module and device state sensing module. The hardware block diagram is shown in Figure 6. Its circuit board and explosion-proof chassis are shown in Figures 7 and 8 .

Figure 6 Structural block diagram of A-type monitoring nodes

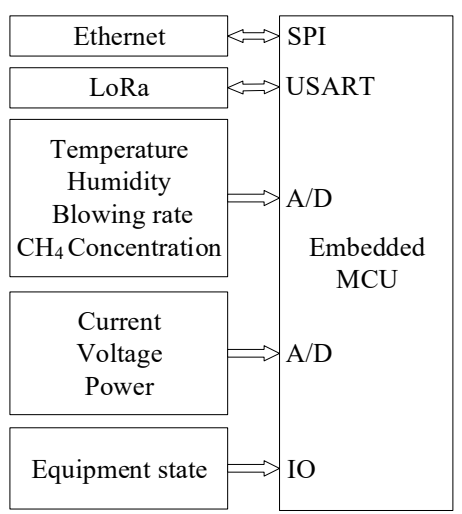

Figure 7 Class A node circuit board (see online version for colours)

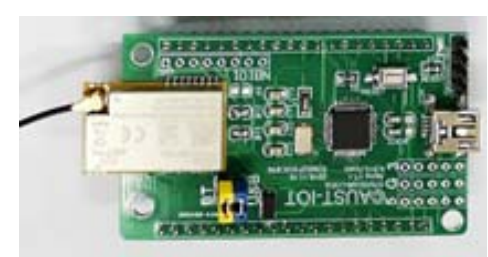

Figure 8 Class A node material object (see online version for colours)

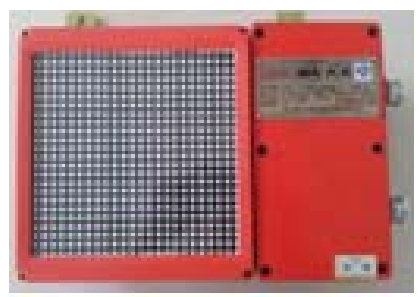

Class B node is the same as for class A node except that it has no access to downhole Ethernet. Class $\mathrm{C}$ nodes can be divided into mine truck carrying and personnel carrying. The type $\mathrm{C}$ nodes carried by mine truck can detect not only many environmental parameters, but also mine truck equipment parameters. The type $\mathrm{C}$ nodes carried by personnel are very light-duty, mainly detecting temperature and humidity, gas environmental parameters and personnel status. Class D nodes are the same as class C nodes carried by people.

In order to verify the design idea proposed in this paper, we selected a 320-metre-long roadway in a mine for industrial experiments. As shown in Figure 9. 
Figure 9 Layout of industrial laboratory opportunities network (see online version for colours)

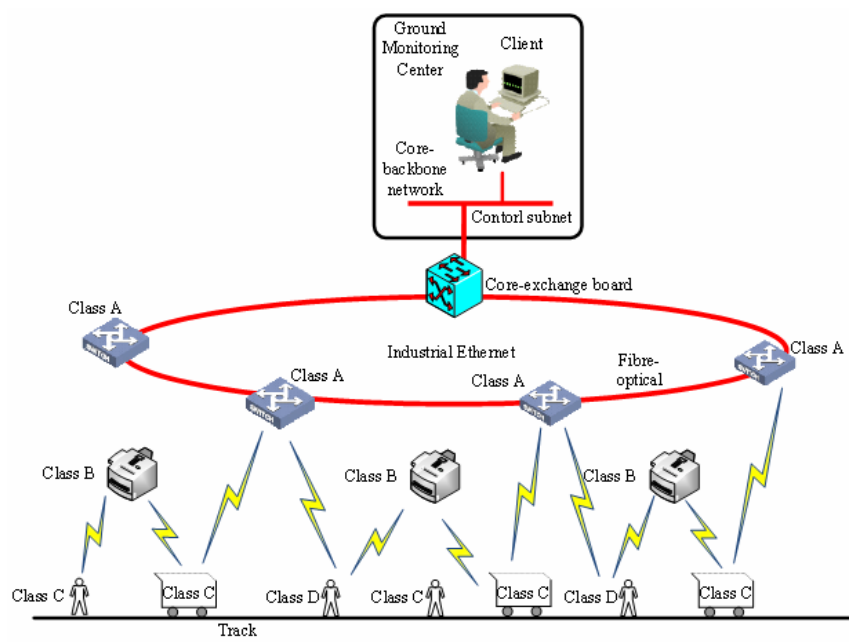

Previously, there were only three A type fixed nodes in this section, as shown in Figure 10. Only environmental information and equipment status within the radius of 5 metres can be collected. The parameters of environment and equipment in other areas have not been collected. Now, based on the three A type fixed nodes (because they have connected to the downhole optical fibre Ethernet, it is tough to add Class A nodes in the downhole environment), we have added six B type fixed nodes (adding nodes is convenient), as shown in Figure 11.

Figure $10320 \mathrm{~m}$ section original class A node layout

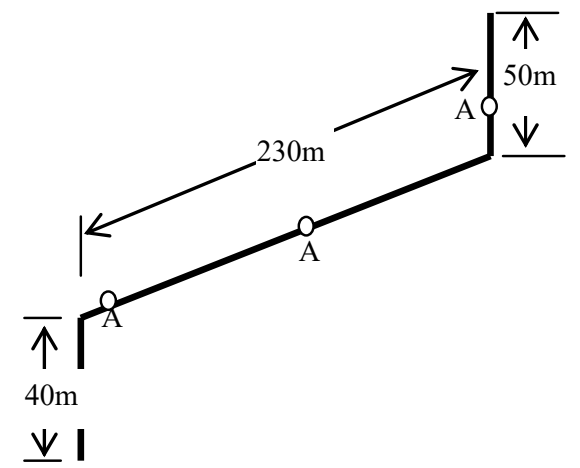

Figure $11320 \mathrm{~m}$ section layout of 'original class A node + new class B node'

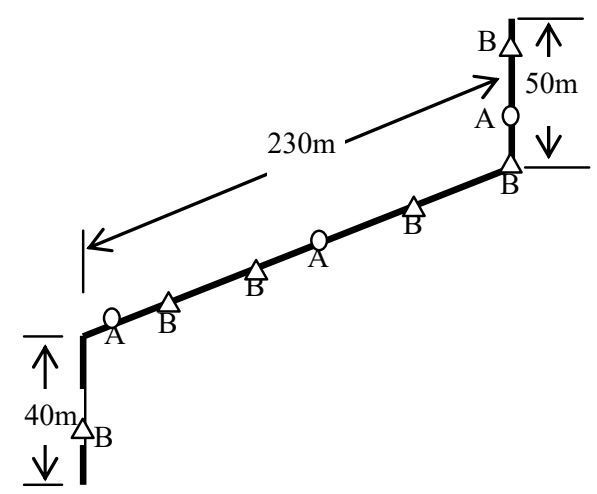

\subsection{Analysis of experimental results}

The first test started at 9 am on 23 November 2018 and ended at 12 noon. Previously, there were only three A class fixed nodes in this section, which could only collect environmental information and equipment status within the radius of 2 metres through the well monitoring system, and the average data uploaded in the first seven days is $63 \mathrm{~kb}$ in the three hours. During the test period, nineteen miners with C-type nodes passed through the test area, and 43 people with mobile nodes passed through the area. B-type nodes and C-type nodes communicate 343 times, and A-type and C-type nodes communicate 182 times. In the meantime, the node status of the $320 \mathrm{~m}$ section at 10:25 am is shown in Figure 12.

Figure 12 Node status of $320 \mathrm{~m}$ section at 10:25 am

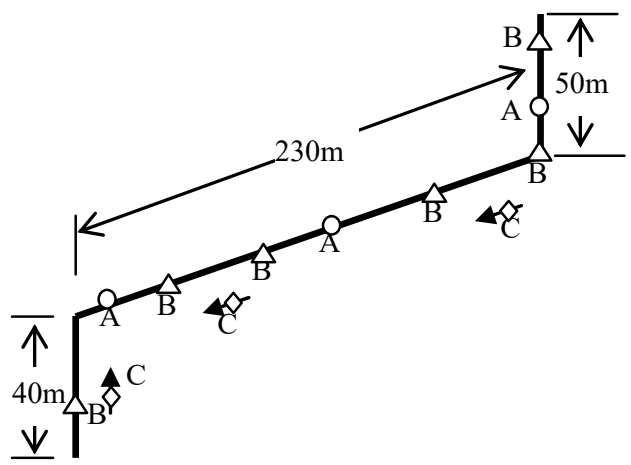

The average update time of regional environmental information detection is 47 seconds, and the maximum update time is 95 seconds. We don't add D-type mobile nodes. The collected information has gas concentration, temperature and humidity, vehicle operation status, main equipment parameters, personnel and other information. So that the coal mines staffs have a comprehensive grasp of the physical information of the region, it provides abundant first-hand information for coal mine safety production. The experimental comparison before and after using this opportunistic routing scheme between 9 am and 12 am shown in Table 2.

Table 2 Experimental comparison of before and after using opportunistic routing schemes at 9:00 to $12: 00 \mathrm{am}$

\begin{tabular}{lcc}
\hline Scheme & $\begin{array}{c}\text { Opportunity } \\
\text { routing is not } \\
\text { adopted }\end{array}$ & $\begin{array}{c}\text { Opportunity } \\
\text { routing is } \\
\text { adopted }\end{array}$ \\
\hline A type node & 3 & 3 \\
A type node & 0 & 6 \\
A type node & 0 & 43 \\
A type node & 0 & 0 \\
Acquisition data & $63 \mathrm{~kb}$ & $2,650 \mathrm{~kb}$ \\
Average update time & $5 \mathrm{~S}$ & $5 \mathrm{~S} / 47 \mathrm{~S}$ \\
Data coverage rate & $<10 \%$ & $>95 \%$ \\
\hline
\end{tabular}


Figure 13 Node status of $320 \mathrm{~m}$ section at 11:40 pm

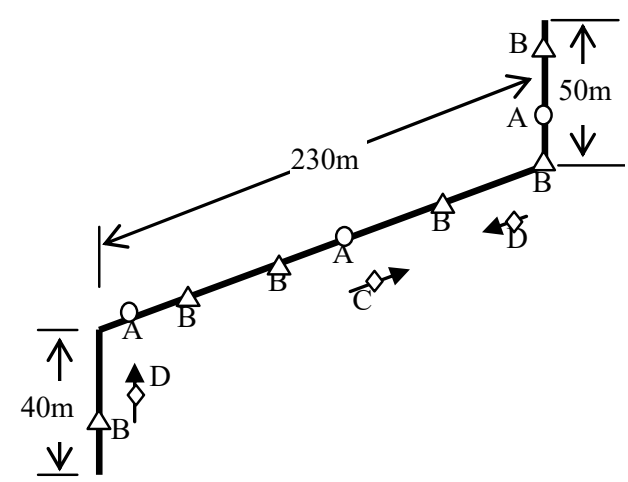

Table 3 Experimental comparison of before and after using opportunistic routing schemes at 10:00 pm to 1:00 am

\begin{tabular}{lccc}
\hline Scheme & $\begin{array}{c}\text { Opportunity } \\
\text { routing is } \\
\text { not adopted }\end{array}$ & $\begin{array}{c}\text { Opportunity } \\
\text { routing is } \\
\text { adopted } \\
\text { (no D type } \\
\text { nodes) }\end{array}$ & $\begin{array}{c}\text { ading is } \\
\text { (added D type } \\
\text { nodes) }\end{array}$ \\
\hline A type node & 3 & 3 & 3 \\
A type node & 0 & 6 & 6 \\
A type node & 0 & 22 & 22 \\
A type node & 0 & 0 & 15 \\
Acquisition data & $63 \mathrm{~kb}$ & $1,850 \mathrm{~kb}$ & $2,590 \mathrm{~kb}$ \\
Average update time & $5 \mathrm{~S}$ & $5 \mathrm{~S} / 70 \mathrm{~S}$ & $5 \mathrm{~S} / 45 \mathrm{~S}$ \\
Data coverage rate & $<10 \%$ & $>90 \%$ & $>95 \%$ \\
\hline
\end{tabular}

We conducted a second experiment from $10 \mathrm{pm}$ to 1 am the next day. The $320 \mathrm{~m}$ section also uploaded $63 \mathrm{~KB}$ of data in that period seven days before that. During this period, thirteen miners carrying C-type nodes passed through the test area, and a total of 22 people carrying mobile nodes passed through the area. D-type mobile nodes were not added. Class $\mathrm{B}$ node and class $\mathrm{C}$ node interact 215 times, Class A node and class $\mathrm{C}$ node interact 127 times. During this period, the average time of detecting and updating regional environmental information is 70 seconds. The longest updating time is 132 seconds. From $10 \mathrm{pm}$ the next night to $1 \mathrm{am}$ the third experiment was conducted according to the proposed dynamic programming strategy for the fourth kind of nodes. Fifteen D-type mobile nodes were added in time-sharing. The node status of the 320 section at 11:40 pm is shown in Figure 13. The average updating time of regional environmental information detection is 40 seconds. The longest updating time is 97 seconds. The experimental comparison of this opportunistic routing scheme before and after $10 \mathrm{pm}$ to $1 \mathrm{am}$ is shown in Table 3. It fully meets the requirements of underground full range monitoring.

\section{Conclusions}

This paper studies the comprehensive utilisation of mine information and the transmission strategy by making full use of mobile resources such as coal mine underground structures and establishing mobile nodes and fixed nodes. In the National Natural Science Foundation of China (51874010), the National Natural Science Foundation of China (61170060), the major innovation platform and the university's innovative talent team_mine IoT R \& D team (2017A053), 2018IRS16 and other project support and Huaibei Mining Group Engineering Testing and Inspection Co., Ltd. Applying the above research results to the underground roadway, track oblique lane and 315 working face of Xutuan Coal Mine of Huaibei Mining Co., Ltd., by using the existing downhole fibre optical fibre Ethernet cable network and four types of nodes, the dynamic full coverage of the above regional information is basically realised.

The most significant advantages of the opportunity routing scheme proposed in this paper are: firstly, it is challenging to arrange class $\mathrm{A}$ nodes in underground coal mine, high cost and inconvenience to add class B nodes, and it is convenient, fast and low cost; secondly, it makes full use of the characteristics of tramcar and personnel movement, realises the full coverage of mine information monitoring, and truly realises the 'material interconnection, intelligent perception, material' in coal mine safety management. As far as the layout of the system itself is concerned, compared with the full coverage of information achieved by all class A nodes, the cost can be reduced by more than $85 \%$. Moreover, due to the current underground conditions, it is difficult to achieve the dense layout of class A joints. Therefore, the opportunistic routing scheme is the best choice for comprehensively perceiving underground coal mines, which provides a strong guarantee for coal mine safety. It has remarkable social and economic benefits.

Next, we will increase the application and promotion of this opportunity's routing results based on industrial laboratories, and make our due contribution to the perception of mines.

\section{Acknowledgements}

This paper has been supported by the National Natural Science Foundation of China (51874010), the National Natural Science Foundation of China (61170060), the major innovation platform, and the university's innovative talent team_mine IoT R\&D team (2017A053) and 2018IRS16. 


\section{References}

Batabyal, S. and Bhaumik, P. (2015) 'Mobility models, traces and impact of mobility on opportunistic routing algorithms: a survey', IEEE Communications Surveys \& Tutorials, Vol. 17, No. 5, p.1.

Borgia, E. (2014) 'The internet of things vision: key features, applications and open issues', Computer Communications, Vol. 54, pp.1-31.

Dhurandher, S.K., Sharma, D.K., Woungang, I. et al. (2013) 'HBPR: history based prediction for routing in infrastructureless opportunistic networks', 2013 IEEE 27th International Conference on Advanced Information Networking and Applications (AINA), pp.931-936.

Ferretti, S. (2013) 'Shaping opportunistic networks', Computer Communications, Vol. 36, No. 5, pp.481-503.

Kerdsri, J. and Veeraklaew, T. (2015) 'Analysis of energy and mobility model on opportunistic routing algorithms', 2015 11th International Conference on Heterogeneous Networking for Quality, Reliability, Security and Robustness (QSHINE), IEEE.

Li, K., Wen, Z., Wang, Z. and Li, S. (2017) 'Optimised placement of wireless sensor networks by evolutionary algorithm', International Journal of Computational Science and Engineering, Vol. 15, Nos. 1-2, pp.74-86.

Li, P., Guo, S., Yu, S. et al. (2014) 'Reliable multicast with pipelined network coding using opportunistic feeding and routing', IEEE Transactions on Parallel \& Distributed Systems, Vol. 25, No. 12, pp.3264-3273.

Liu, Y., Zhang, Q. and Ni, L. (2010) 'Opportunity-based topology control in wireless sensor networks', IEEE Transactions on Parallel \& Distributed Systems, Vol. 21, No. 3, pp.405-416.

Nguyen, A.D., Senac, P. and Diaz, M. (2015) 'Modelling mobile opportunistic networks - from mobility to structural and behavioural analysis', Ad Hoc Networks, Vol. 24, pp.161-174.

Pásztor, B., Musolesi, M. and Mascolo, C. (2007) 'Opportunistic mobile sensor data collection with SCAR', IEEE International Conference on Mobile Adhoc and Sensor Systems Conference, pp.1-12, IEEE.

Qin, Q., He, Y.Q. and Nie, L.M. (2015) 'Cooperative cloud service aware mobile internet coverage connectivity guarantee protocol based on sensor opportunistic coverage mechanism', Journal of Electrical \& Computer Engineering, Vol. 2015, No. 1, p.5.

Sasirekha, V., Chandrasekar, C. and Ilangkumaran, M. (2015) 'Heterogeneous wireless network vertical handoff decision using hybrid multi-criteria decision-making technique', International Journal of Computational Science and Engineering, Vol. 10, No. 3, pp.263-280.
Sitanayah, L., Datta, A. and Cardell-Oliver, R. (2010) 'Heuristic algorithm for finding boundary cycles in location-free low density wireless sensor networks', Computer Networks, Vol. 54, No. 7, pp.1630-1645.

Soares, V.N.G.J., Rodrigues, J.J.P.C. and Farahmand, F. (2014) 'GeoSpray: a geographic routing protocol for vehicular delay-tolerant networks', Information Fusion, Vol. 15, No. 1, pp.102-113.

Trifunovic, S., Kouyoumdjieva, S.T., Distl, B. et al. (2017) 'A decade of research in opportunistic networks: challenges, relevance, and future directions', IEEE Communications Magazine, Vol. 55, No. 1, pp.168-173.

Wang, S., Wang, X., Huang, J. et al. (2015) 'The potential of mobile opportunistic networks for data disseminations', IEEE Transactions on Vehicular Technology, Vol. 65, No. 2, p.1.

Wu, D., Cao, J., Ling, Y. et al. (2012) 'Routing algorithm based on multi-community evolutionary game for VANET', Journal of Networks, Vol. 7, No. 7.

Xu, Z., Zhang, S., Choo, K-K.R., Mei, L., Wei, X., Luo, X., Hu, C. and Liu, Y. (2017) 'Hierarchy-cutting model based association semantic for analyzing domain topic on the web', IEEE Trans. Industrial Informatics, Vol. 13, No. 4, pp.1941-1950.

Yang, S., Adeel, U., Tahir, Y. et al. (2016) 'Practical opportunistic data collection in wireless sensor networks with mobile sinks', IEEE Transactions on Mobile Computing, Vol. 16, No. 5, p.1.

Zhang, S., Wei, Z., Wang, Y. and Liao, T. (2018) 'Sentiment analysis of Chinese micro-blog text based on extended sentiment dictionary', Future Generation Computer Systems, Vol. 81, pp.395-403.

Zhang, Y., Yang, W., Han, D. et al. (2014) 'An integrated environment monitoring system for underground coal mines wireless sensor network subsystem with multi-parameter monitoring', Sensors, Vol. 14, No. 7, pp.13149-13170.

Zhao, C., Liu, F. and Hai, X. (2013) 'An application of wireless sensor networks in underground coal mine', International Journal of Future Generation Communication and Networking, Vol. 6, No. 5, pp.117-126.

Zhao, D., Ma, H., Liu, L. et al. (2015) 'Opportunistic coverage for urban vehicular sensing', Computer Communications, Vol. 60, No. C, pp.71-85.

Zhou, Y. and Peng, D. (2018) 'A new model of vehicular ad hoc networks based on artificial immune theory', International Journal of Computational Science \& Engineering, Vol. 16, No. 2, pp.153-161. 